\title{
Quadriparesis caused by retropharyngeal and epidural abscess in COVID-19 patients
}

\author{
Nicolò Gennaro ${ }^{1,2} \cdot$ Cristiana Bonifacio $^{2} \cdot$ Manuel Corato $^{3} \cdot$ Davide Milani $^{4} \cdot$ Letterio S. Politi $^{1,5,6} \mathbb{C}_{\mathbb{C}}$
}

Received: 25 January 2021 / Accepted: 1 March 2021 / Published online: 6 March 2021

(C) Fondazione Società Italiana di Neurologia 2021

Dear editor,

Coronavirus disease 2019 (COVID-19) was declared a pandemic by the World Health Organization (WHO) on March 11, 2020. At the time, Italy was already one the most affected country in Europe and on May 25 accounts for more than 230,000 positive cases and 33,000 deaths [1]. COVID-19 involvement is mainly confined to the lungs, but also, extrapulmonary manifestations have recently been described [2] Among the latter, neurological manifestations are also emerging. Opportunistic and secondary infections also are reported to be common in patients diagnosed with COVID-19[3]. Retro-pharyngeal abscesses extending into the epidural space and causing spinal cord compression and quadriparesis are exceptional. They are often secondary to lesions caused by foreign bodies, or related to Staphylococcus aureus pharyngitis in immunocompromised patients [4]. Here we report two of such cases who were admitted in the Emergency Department (ED) of our institution during the first 2 months, who were positive for SARS-CoV-2 infections. A brief discussion about the possible etiopathogenesis of this correlation is then provided.

Letterio S. Politi

letterio.politi@hunimed.eu

1 Department of Biomedical Sciences, Humanitas University, Via Rita Levi Montalcini 4, 20090, Pieve Emanuele Milan, Italy

2 Radiology Department, IRCCS Humanitas Research Hospital, Rozzano Milan, Italy

3 Neurology Department, IRCCS Humanitas Research Hospital, Rozzano Milan, Italy

4 Neurosurgery Department, IRCCS Humanitas Research Hospital, Rozzano Milan, Italy

5 Neuroradiology Department, IRCCS Humanitas Research Hospital, Rozzano Milan, Italy

6 Radiology Department and Hematology \& Oncology Division, Boston Children's Hospital, Boston, MA, USA

\section{Case 1}

A 56-year-old man was admitted to the ED for the sudden onset of quadriparesis. The patient had been experiencing sore throat and neck pain radiating along his right arm for 2 days. One week before, he had been discharged from a nearby hospital where he had received treatment for COVID-19, and was currently spending his quarantine in a dedicated facility. During his prior hospitalization, as non-CPAP responsive, he required endotracheal intubation and multidrug therapy including hydroxychloroquine, antibiotics, corticosteroids, and off-label therapy with tocilizumab.

His medical history was otherwise unremarkable. Neurologic physical examination revealed asymmetric quadriparesis, more pronounced on the left side. Deep tendon reflexes were diminished in the upper extremities and increased in the lower limbs. Ankle clonus and Babinski's sign were bilaterally present. Lhermitte's sign was clearly positive and no sensory level was identified. The temperature at admission was $38.2{ }^{\circ} \mathrm{C}$. The laboratory showed neutrophilic leukocytosis $\left(14.000 / \mathrm{mm}^{3}\right)$, high levels of C-reactive protein $(37 \mathrm{mg} / \mathrm{L})$, anemia $(102$ $\mathrm{g} / \mathrm{L})$, and reduced peripheral blood saturation $\left(\mathrm{S}_{2} 91 \%\right)$. Non-contrast chest CT showed persistence of bilateral lung parenchymal alterations compatible with the recent SARS-Cov-2 infection. Since cervical myelitis was suspected, the patient underwent a contrast-enhanced MRI of the cervical and thoracic spine. An extensive retropharyngeal abscess was observed, with epidural extension from level $\mathrm{C} 4$ to $\mathrm{C} 6$, displacing posteriorly to the right and compressing the cord (Fig. 1). Initial signs of compressive myelopathy, visualized as poorly defined hyper-intensity on T2-weighted images within the cord, were also observed. No signs of spondylodiscitis were observed. On the same day, urgent decompressive cervical laminectomy was performed to prevent irreversible neurological damage. Cultures resulted positive for Staphylococcus aureus infection. 


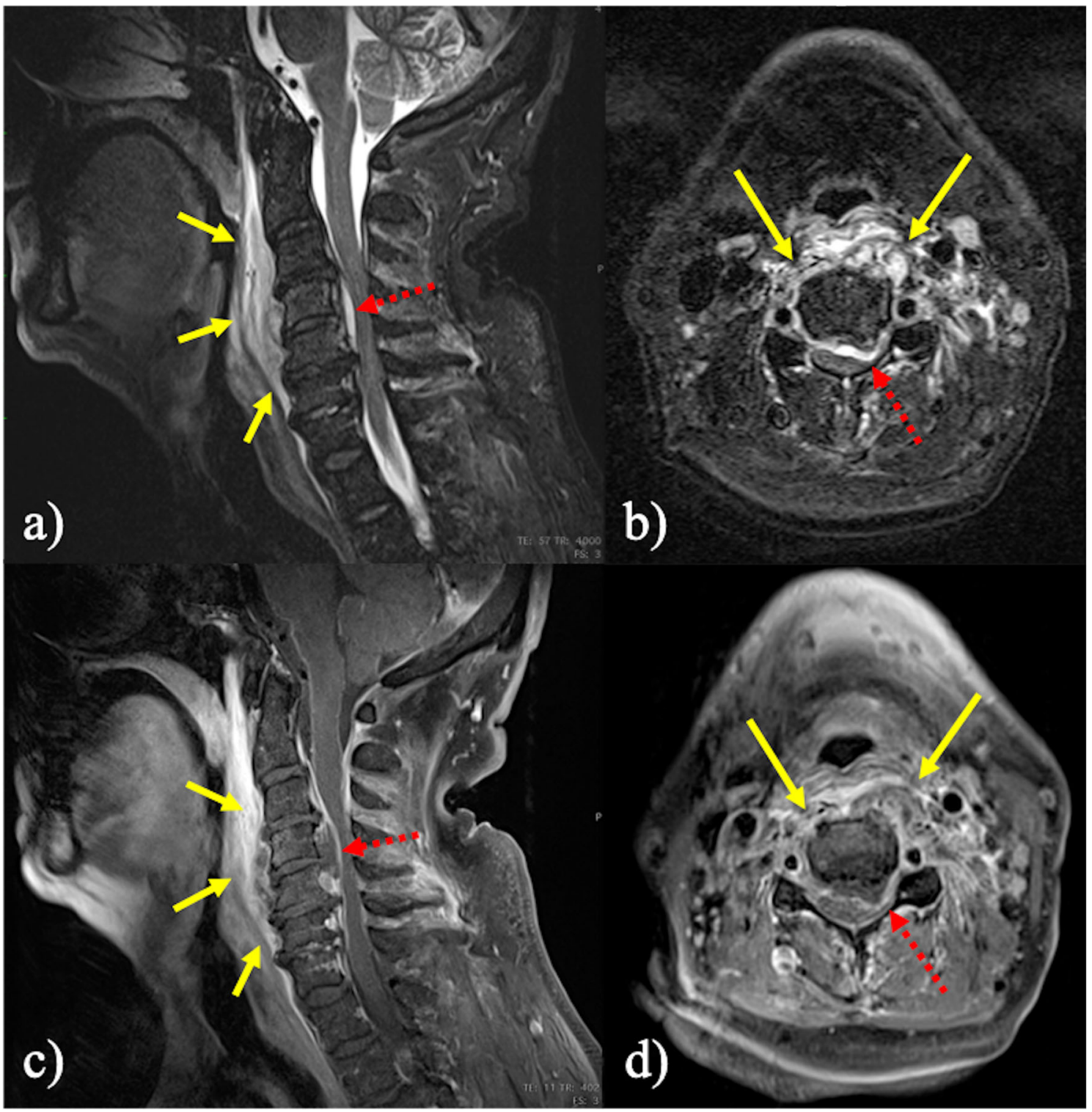

Fig. 1 Cervical spine MRI showing retro-pharyngeal and epidural abscess, causing spinal cord compression (Case 1) a sagittal and b axial short-tau inversion recovery (STIR) T2-weighted images, and c sagittal and $\mathbf{d}$ axial post-contrast fat-saturated $\mathrm{T} 1$-weighted images demonstrating

\section{Case 2}

A 55-year-old man was admitted to the ED for the onset of neck pain followed by decreased strength in lower limbs. Five days earlier, he had been discharged from a nearby hospital where he was treated for COVID-19 with CPAP non-invasive ventilation. He was discharged with a negative nasopharyngeal swab and addressed to quarantine.

His medical history was positive for chronic hypertension and STEMI treated by angioplasty 3 months before. Neurologic physical examination revealed asymmetric quadriparesis with loss of strength in both the proximal lower limbs and the distal upper limbs, especially in the right hemi-body. Deep tendon a large retro-pharyngeal abscess (yellow arrows) extending in the epidural space (dotted red arrows) and causing cord displacement and compression

reflexes were diminished in the upper and lower extremities. Babinski's test turned positive bilaterally. The patient was complaining of paresthesiae in both hands, and a partially sensitive D5 level was identified.

The temperature at admission was $37.6{ }^{\circ} \mathrm{C}$. The laboratory showed neutrophilic leukocytosis $\left(11.700 / \mathrm{mm}^{3}\right)$, lymphopenia $\left(400 / \mathrm{mm}^{3}\right)$, high levels of C-reactive protein $(211 \mathrm{mg} / \mathrm{L})$, anemia (112 g/L), and normal peripheral blood saturation ( $\left.\mathrm{SO}_{2} 99 \%\right)$. The patient underwent a contrast-enhanced MRI of the cervical and thoracic spine. A retro-pharyngeal abscess with epidural extension from level C5 to C7 was identified, displacing and compressing the cord, particularly on the right side. No signs of myelopathy were described. The day after 
admission, decompressive cervical laminectomy was performed. Cultures resulted positive for methicillin-resistant Staphylococcus aureus infection.

\section{Discussion}

Here we describe two cases of COVID-19 patients presenting retro-pharyngeal and epidural abscess from Staphylococcus aureus who required urgent decompressive surgery. In Case 1, the patient had been intubated and treated with off-label use of IL-6 receptor blockade agent (tocilizumab). Cytokine release storm (CRS) seems an important player in the lethal pathogenesis of the novel coronavirus disease. As a consequence, immunemodulating drugs, including tocilizumab, are being employed as off-label medications in the treatment of COVID-19, showing some efficacy [5]. The rationale for the employment of such immune-modulating drugs in the COVID-19 is to suppress immunologic response and break the CRS. It is important to recall that such drugs expose patients to the risk of developing serious infections. In this regard, a case of retro-pharyngeal abscess in a 62-year-old patient receiving immunomodulation therapy for rheumatoid arthritis was reported by Kakarala $\mathrm{K}$ et al. [6]. Neurologic signs like quadriparesis are an exceedingly rare clinical presentation of a retro-pharyngeal abscess, with only two reports describing an extension into the epidural spaces and neurologic deficits [7, 8]. Decompressive laminectomy, debridement, and evacuation of infected tissues represent the treatment of choice and must be followed by specific culture-guided intravenous antibiotics for 4-6 weeks. Since a strong correlation exists between the duration of symptoms prior to surgery, and the degree of permanent neurologic deficits, timely surgical intervention is imperative [9]. Conversely, the patient described in Case 2 did not receive any drug therapy for Covid-19 but CPAP noninvasive ventilation only. This suggests that immunosuppression may not necessarily be related to the immunomodulation therapy, but might possibly be related to the immunosuppressive effect of the SARS-Cov-2 infection. SARS-CoV-2 is known to cause lymphopenia, while bacterial abscesses are usually related to neutropenia. However, in COVID-19 patients, secondary bacterial or opportunistic infections were reported in up to $27 \%$ of hospitalized patients in Wuhan, China [10]. Therefore, the possibility of infectious complications resulting in surgical emergencies should be considered regardless of the treatments received in COVID-19 patients. Further studies on larger populations are needed to typify opportunistic infections in COVID-19 patients after discharge and understand the need for follow-up of patients recovered from COVID-19.

Author contribution NG and CB wrote the draft. MC provided neurological care. DM performed the surgery. LSP conceptualized, supervised, and reviewed the draft.

\section{Declarations}

Ethics approval and consent to participate The need for approval was waived for this case report. All procedures performed in studies involving human participants were in accordance with the ethical standards of the institutional and/or national research committee and with the 1964 Helsinki declaration and its later amendments or comparable ethical standards. This article does not contain any studies with human participants or animals performed by any of the authors. Written informed consent was obtained from the individual participant of the study to use clinical data and medical images.

Consent for publication The individual participant of the study gave written consent prior publication.

Conflict of interest The authors declare no competing interests.

\section{References}

1. Covid-19 - Situazione in Italia. http://www.salute.gov.it/portale/ nuovocoronavirus/dettaglioContenutiNuovoCoronavirus.jsp?area= nuovoCoronavirus \&id $=5351 \&$ lingua $=$ italiano $\&$ menu $=$ vuoto. Accessed 25 Dec 2020

2. Behzad S, Aghaghazvini L, Radmard AR, Gholamrezanezhad A (2020) Extrapulmonary manifestations of COVID-19: Radiologic and clinical overview. Clin Imaging 66:35-41. https://doi.org/10. 1016/j.clinimag.2020.05.013

3. Clancy CJ, Nguyen MH (2020) COVID-19, superinfections and antimicrobial development: What can we expect? Clin Infect Dis. https://doi.org/10.1093/cid/ciaa524

4. Liou J-H, Su Y-J (1543) (2015) Unusual cervical spine epidural abscess. Am J Emerg Med 33:e1-e2. https://doi.org/10.1016/j.ajem.2015.07.052

5. Zhang $\mathrm{C}, \mathrm{Wu} \mathrm{Z}, \mathrm{Li} \mathrm{J}-\mathrm{W}$ et al (2020) Cytokine release syndrome in severe COVID-19: interleukin-6 receptor antagonist tocilizumab may be the key to reduce mortality. Int J Antimicrob Agents 55: 105954. https://doi.org/10.1016/j.ijantimicag.2020.105954

6. Kakarala K, Durand ML, Emerick KS (2010) Retropharyngeal abscess in the setting of immune modulation for rheumatoid arthritis. Laryngoscope 120(Suppl 4):S131. https://doi.org/10.1002/lary.21595

7. Koopmann CF, Miller RW, Coulthard SW (1984) Retropharyngeal abscess associated with progressive quadriplegia, an epidural abscess, renal failure. and jaundice. Otolaryngol Head Neck Surg 92: 114-118. https://doi.org/10.1177/019459988409200124

8. Jalisi S, Sakai O, Jamal BT, Mardirossian V (2017) Features of prevertebral disease in patients presenting to a head and neck surgery clinic with neck pain. Ann Maxillofac Surg 7:228-231. https:// doi.org/10.4103/ams.ams_54_17

9. Thomson C (2018) Spinal cord compression secondary to epidural abscess: the importance of prompt diagnosis and management. BMJ Case Rep. https://doi.org/10.1136/bcr-2017-220694

10. Zhou F, Yu T, Du R et al (2020) Clinical course and risk factors for mortality of adult inpatients with COVID-19 in Wuhan, China: a retrospective cohort study. Lancet 395:1054-1062. https://doi.org/ 10.1016/S0140-6736(20)30566-3

Publisher's note Springer Nature remains neutral with regard to jurisdictional claims in published maps and institutional affiliations. 